\title{
Pressure blindness and the interocular transfer of size aftereffects
}

\author{
GLENN E. MEYER \\ State University of New York at Buffalo, 4230 Ridge Lea Road, Amherst, New York 14226
}

\begin{abstract}
After observation of a stimulus composed of a top grating with large bar widths (low spatial frequency) and a bottom grating of narrow lines (high spatial frequency), a subsequently presented test grating of medium bar width appears to have a higher spatial frequency on the top half than on the bottom. Although this size aftereffect can be obtained dichoptically, this does not necessarily imply a central locus, since retinal input from the adapted eye could produce the effect. Ss were tested for the aftereffect in the adapted eye and for interocular transfer with and without pressure blinding the adapted eye. In this last condition, input from the adapted eye cannot reach the cortex. However, the aftereffect was equally present under all three conditions. This result suggests that size and frequency adaptation have a central locus.
\end{abstract}

After prolonged exposure to a grating with a large bar width (low spatial frequency), the bars in a subsequently presented medium-width grating appear even thinner. That is, there is an apparent increase in the grating's spatial frequency. Similarly, adaptation to gratings of high spatial frequency produces an apparent decrease in the spatial frequency of the medium bar width grating. The reader can verify this by scanning the center bar of Grating $A$ in Fig. 1 for the $60 \mathrm{sec}$ and then fixating the center bar of Grating B. Blakemore and Sutton (1969) suggest that this phenomenon is due to the adaptation of cortical size- and spatial frequency-specific detectors. They propose that after adaptation to the low spatial frequency grating there is decreased responsiveness of the "wide" end of the collection of neural channels that would normally respond to the medium-sized lines. This causes the lines to appear thinner. Using the same logic, adaptation to narrow lines causes an apparent decrease in the spatial frequency of lower frequency gratings. The effect is assumed to be central, because populations of neurons sensitive to size and spatial frequency are found in the cortex and because it transfers interocularly (Blakemore, Nachmias, \& Sutton, 1970). In primates, the inputs from the two eyes do not converge before the cortex (Isaacson, Douglas, Lubar, \& Schmaltz, 1971).

However, interocular transfer does not necessarily prove that the locus is cortical, since inputs from the adapted eye may combine with those from the unadapted eye to produce an interocular effect (Day, 1958; Julesz, 1971). For example, a positive red afterimage in one eye can be fused with a green disk subsequently presented to the other eye. The disk will now appear to be a desaturated yellow. However, Ss who are pressure-blinded in the eye in which the afterimage is produced show no change in the perception of a green disk presented to the other eye (Gestrin \& Teller, 1969). Pressure blinding reduces the blood supply to the retina and produces the sensation of subjective blindness. Visual evoked cortical potentials cannot be elicited from an eye pressure-blinded to the criterion of subjective blindness (Fox, Blake, \& Bourne, 1973). Since it is unlikely that impulses from the bleached eye reached the cortex, Gestrin and Teller's results would seem to indicate that the eftereffect is retinally based.

The size aftereffect or frequency shift (hereafter called SAE) may similarly be due to retinal adaptation. After adapting to wide lines, the retinal
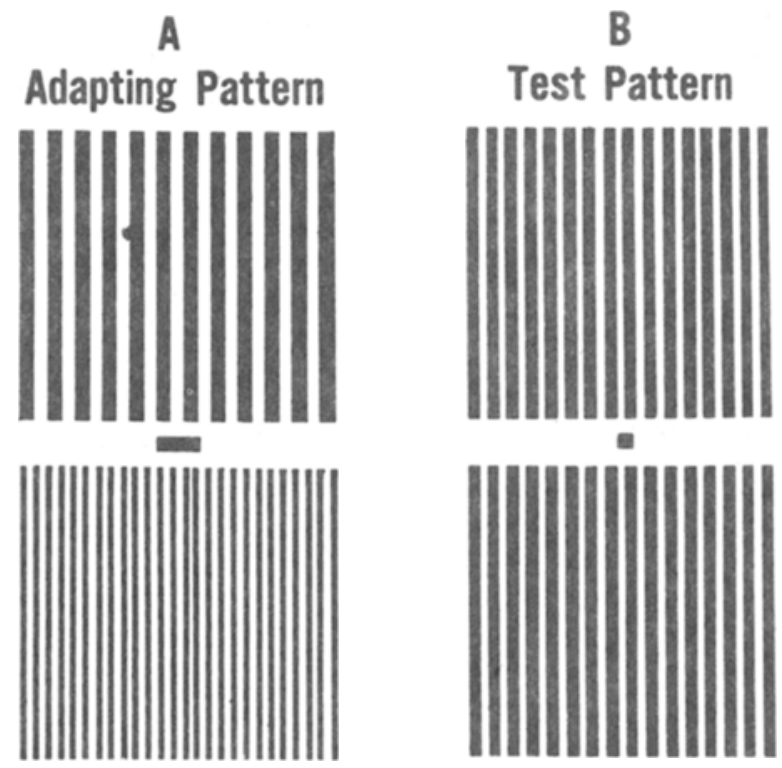

Fig. 1. Example of spatial frequencies used for adapting and test pattems. 
ganglion cells corresponding to the pattern would be fatigued. Responses from the unaffected elements in either site might indicate the presence of narrow lines (somewhat analogously to negative afterimages), and these would be sent to the cortex and fused with the test pattern presented to the unadapted eye. This could conceivably produce an interocular transfer of the SAE.

The present study sought to examine the locus of the SAE by using interocular transfer in conjunction with pressure blindness. Ss were adapted in one eye to a display similar to that used by Blakemore and Sutton (1969) for producing SAEs. They were then tested for the aftereffect (1) Condition A-in the adapted eye, (2) Condition $U$-in the unadapted eye under normal conditions, and (3) Condition UB-in the unadapted eye while the adapted eye was pressure-blinded. Under Condition UB, it was highly unlikely that meaningful impulses from the adapted eye could reach the cortex, as the blood supply to the retinal ganglion cells was cut off. If, for Condition UB, there was no interocular transfer, or if there was a significant reduction in the number of Ss reporting the aftereffect as compared with Conditions $U$ and $A$, then one might infer low-level involvement in spatial frequency adaptation. If the SAE was as strong under Condition UB as under the other conditions, this would indicate that the effect was central.

\section{METHOD}

\section{Subjects}

Twelve women participated in the study (10 undergraduate and 2 graduate). All were naive as to the purpose of the experiment and the nature of the visual aftereffect. The Ss were all volunteers, and the pressure-blinding technique was explained to them before the experiment started.

\section{Materials}

The adaptation slide consisted of two vertical square-wave gratings separated by a fixation bar. The upper grating (designated "wide") was $2.2 \mathrm{cycles} / \mathrm{deg}$; the lower grating (designated "narrow") was 4.8 cycles $/ \mathrm{deg}$. In the test slide, the two vertical halves both had a spatial frequency $(3.2 \mathrm{cycles} / \mathrm{deg})$ intermediate to those of the adaptation slide. Each slide subtended $3.5 \mathrm{deg}$ vertically $x 5$ deg horizontally, with each grating being $1.6 \times 5 \mathrm{deg}$, with $.28 \mathrm{deg}$ separation. The slides were presented in a Scientific Prototype (Model GB) three-channel tachistoscope. Mean luminance of the adaptation slide was $2.42 \mathrm{fL}$, while the test luminance was $1.6 \mathrm{fL}$. Lower test luminance has been found to maximize the perception of visual aftereffects (Blakemore, Muncey \& Ridley, 1971; Parker, 1972). The contrast ratio for all stimuli were .97 (contrast $\left.=I_{\max }-I_{\min } / I_{\max }+I_{\min }\right)$. Black cardboard masks were used to block the tachistoscope's right eyepiece during adaptation.

\section{Procedure}

As each $S$ was to be adapted in the left eye, practice was given in pressure-blinding that eye for subsequent testing trials. A blank stimulus field was presented and she was instructed to press against the left side of her eye, while keeping the right eye closed, until the blank field disappeared from view. When this happened, she was to open her right eye and fixate the field with it. $S$ was told to be sure to keep her left eye open during the application of pressure, and the
E watched to make sure this was done. The entire procedure took approximately $30 \mathrm{sec}$, and duplicated the UB test trial described below except for the lack of presentation of the test slide. The practice sequence was repeated until $S$ felt comfortable with the procedure and thought she could do it on command during testing.

Next came preadaptation exposures of the test slide to uncover any existing response biases. The equal-frequency test slide was presented to the left eye, which was to be used for adaptation (Condition A), the unadapted right eye, which was to be used for testing interocular transfer (Condition $\mathrm{U}$ ), and the right eye while the left eye was pressure-blinded (Condition UB(. Under all conditions, a test trial consisted of fixating the center dot of the test slide and reporting one of three possible responses given before testing started: (1) top half lines appear wider than bottom half, (2) both halves appear equal, and (3) bottom lines appear wider than top half. In Conditions $A$ and $U$, the eye not used for observation was kept closed. For Condition UB tests, a blank field was presented in one channel of the tachistoscope to the left eye while the right eye was kept closed. The $S$ was told to pressure-blind her left eye. When the field faded, the $S$ reported this to the $E$, who then switched off the blank field and turned on the test stimulus in another channel. The $S$ meanwhile kept pressing her left eye to maintain the facing and opened her right eye to observe the test pattern.

Then the $S$ was adapted in the left eye with 10 cycles of a 50 -sec exposure to the unequal frequency slide alternated with $4 \mathrm{sec}$ of darkness. Total grating adaptation time was $500 \mathrm{sec}$. Alternation of the grating and darkness was used to relieve the S's tedium during adaptation. During this period, the right eyepiece was blocked with cardboard. When the adaptation slide was on, the $S$ was told to keep her fixation on the center line to assure proper retinal localization of the different frequencies.

After adaptation, each $S$ was retested with the test slide. The testing sequence used here and for pretests was the following: Each $S$ made two observations under each of the three test conditions ( $A$, $U, U B$ ) for a total of six observations per $S$. With three test conditions and two trials of each, there are six counterbalanced orders of test conditions (for example, A, UB, U, U, UB, A; U, UB, A, A, UB, $U$; etc.). Each sequence was administered to 2 ss for a total of $12 \mathrm{Ss}$. The entire testing procedure took approximately $2 \mathrm{~min}$. The duration of the adaptation period was presumed to be long enough for the aftereffect not to greatly decrease over testing trials (see Blakemore et al, 1970, p. 745, Fig. 10).

\section{RESULTS}

During pretesting, all $S$ s reported that the test pattern halves were equal in size under all conditions (Table 1). According to Blakemore and Sutton (1969) and Blakemore et al (1970), adapting to Pattern $A$ in Fig. 1 causes the top half of the equal-frequency test pattern (B) to appear to be of higher spatial frequency than the bottom. This, in fact, occurred with the test

Table 1

Mean Percentage of Size Aftereffect Report Under Conditions $A, U$, and UB Averaged Across Twelve Ss, Two Trials in Each Condition

\begin{tabular}{|c|c|c|c|c|c|c|}
\hline & \multicolumn{6}{|c|}{ Conditions* } \\
\hline & \multicolumn{2}{|c|}{ A } & \multicolumn{2}{|c|}{ UB } & \multicolumn{2}{|c|}{$\mathbf{U}$} \\
\hline & Mean & $\mathrm{SD}$ & Mean & SD & Mean & SD \\
\hline Preadaptation $\dagger$ & 0 & & 0 & & 0 & \\
\hline Postadaptation & 75.0 & 38.2 . & 70.8 & 38.0 & 75.0 & 32.3 \\
\hline
\end{tabular}

${ }^{*} A=$ adapted eye, $U B=$ unadapted eye while adapted eye is pressure blinded, $U=$ unadapted eye.

tNo SDs are reported for preadaptation conditions, as all scores were 0 . 
slide. Ss either reported the SAE or said that the halves were equal. No reversals of the expected aftereffect were recorded. The data were thus analyzed on the basis of aftereffect absence or presence ( 0 or 1 , respectively). The mean percentages of aftereffect report based on the two observations for each condition for all Ss are presented in Table 1. Three independent $t$ tests were performed with a hypothesized population mean of 0 (no aftereffect). With $11 \mathrm{df}$ for each group, the $\mathrm{t}$ values of the A, UB, and $U$ postadaptation reports were $6.51,6.17$, and 7.71 , respectively ( $p<.01$ for all conditions). The SAE is strongly present.

An analysis of variance for a randomized block factorial design (Kirk, 1968) was performed on the postadaptation data to look for differences between condition means (such analysis is appropriate for dichotomous data; see Cochran, 1950). There were no significant differences between conditions $[F(2,22)<$ 1]. In fact, the lower mean for Condition UB is due to only one less positive aftereffect observation. The differences between Ss were significant $[F(11,22)=$ $2.78, \mathrm{p}<.05)$, including those who saw no aftereffects and those who did.

It could be argued that Ss who initially received Conditions A or U might be biased on subsequent UB trials to report the SAE even if it were not present. However, on the first trial, one would assume no such bias to be operating. There were four Ss who received each condition, $U B, U$, or $A$, as the first test. The mean report percentages for thes subgroups were 75 , 75 , and 100 , respectively. There were no differences between $U B$ and $U$ subgroups, and no significant differences between U or UB vs A [for both, $t(6)=$ $1.0, p>.20]$. With response bias minimized, there still seem to be no differences between $U B, U$, and $A$ conditions.

\section{DISCUSSION}

The presence of the SAE, tested interocularly, was not affected by presure-blinding the adapted eye. With no meaningful impulses reaching the cortex from the adapted eye, the adaptation must have a central locus, presumably in size- and/or frequencysensitive detectors, as Blakemore and Sutton (1969) and Blakemore et al (1970) originally stated.

There are other sets of data that support the hypothesis that size- and frequency-specific adaptation do not occur in the retina. Pressure blinding of the adapted eye does not influence the interocular transfer of movement aftereffects (Barlow \& Brindley, 1963; Scott \& Wood, 1966) or of spatial frequencyspecific threshold increments (Blake \& Fox, 1972). Further, Graham (1972) found that illuminance and pattern drift conditions that would alter ganglion cell sensitivity do not affect spatial frequency sensitivity functions, and concludes that neural units higher than the retinal ganglion cell level determine spatial frequency channels. These data are supported by the present results, which demonstrate interocular transfer of the SAE despite pressure-blinding of the adapted eye.

\section{REFERENCES}

BARLow, H. B., \& BRindley, G. S. Interocular transfer of movement aftereffects during pressure blinding of the stimulated eye. Nature, 1963, 200, 1347.

Blake, R., \& Fox, R. Interocular transfer of adaptation to spatial frequency during retinal ischaemia. Nature-New Biology, 1972, 240, 76-77.

Blakemore, C., Muncey, J. P. J., \& Ridley, R. M. Perceptual fading of stabilized cortical image. Nature, 1971, 223, 204-205.

Blakemore, C., Nachmias, J., \& Sutton, P. The perceived spatial frequency shift: Evidence for frequency-selective neurones in the human brain. Journal of Physiology, 1970, 210. 727.750.

Blakemore, C., \& Sutton, P. Size adaptation: A new aftereffect. Science, 1969, 166, 245-247.

Cochran, W. G. Comparisons of percentages in matched samples. Biometrika, 1950, 37, 256-266.

DAY, R. H. On interocular transfer and the central origin of visual aftereffects. American Journal of Psychology, 1958, 71, 784-790.

Fox, T., Blake, R., \& Bourne, J. R. Visual evoked cortical potentials during pressure blindness. Vision Research, 1973, 13, 501-503.

Gestrin, P. J., \& Teleer, D. Interocular hue shifts and pressure blindness. Vision Research, 1969, 9, 1267-1271.

Graham, N. Spatial frequency channels in the human visual system: Effects of luminance and pattern drift rate. Vision Research, 1972, 12, 53-68.

Isaacson, R. L., Douglas, R. L., Lubar, J. F., \& Schmaltz, L. W. A primer of physiological psychology. New York: Harper \& Row, 1971 .

Julesz, B. Foundations of cyclopean perception. Chicago: University of Chicago Press, 1971.

KIRK, R. E. Experimental design: Procedures for the behavioral sciences. Belmont, California: Brooks/Cole, 1968.

PARKER, D. M. Contrast and size variables in the tilt aftereffect. Quarterly Journal of Experimental Psychology, 1972, 24, 1-7.

ScotT, T. R., \& Wood, D. Z. Retinal anoxia and the locus of the aftereffect of motion. American Journal of Psychology, 1966, $79,435-442$.

(Received for publication June 22, 1973; revision received April 19, 1974.) 